\title{
Double-Stranded RNA-Specific Adenosine Deaminase
}

National Cancer Institute

\section{Source}

National Cancer Institute. Double-Stranded RNA-Specific Adenosine Deaminase. NCI

Thesaurus. Code C157285.

Double-stranded RNA-specific adenosine deaminase (1226 aa, 136 kDa) is encoded by the human ADAR gene. This protein plays a role in the deamination of adenosine to inosine in double-stranded RNA (A-to-I RNA editing). 